\title{
Die elektronenmikroskopische Darstellung des Mosaikvirus der Zuckerrüben
}

\author{
Von Ferdinand Schneider und Karl-Wolfgang Mundry \\ Aus dem Institut für landwirtschaftliche Technologie und Zuckerindustrie \\ an der Technischen Hochschule, Braunschweig \\ (Z. Naturforschg. 11 b, 393-394 [1956]; eingegangen am 11. Mai 1956)
}

Elektronenmikroskopischer Abbildung zufolge ist ein $700 \mathrm{~m} \mu$ langes, stäbchenförmiges Partikel als Erreger der Mosaikkrankheit der Rüben anzusehen.

$\mathrm{I}$

m Gegensatz zum Vergilbungsvirus der Rüben ${ }^{1,2,3}$ fehlten bislang noch elektronenmikroskopische $\mathrm{Ab}$ bildung und Vermessung des mit diesem Virus häufig vergesellschafteten Mosaikvirus der Rüben (RMV). Die hier mitgeteilte Untersuchung hatte nicht nur die elektronenmikroskopische Darstellung des RMV zum Ziel, sondern auch die elektronenmikroskopische Kontrolle der auf manchen Wirtspflanzen sichtbaren primären Infektionsherde. Dadurch sollte die Sicherheit der Identität evtl. aufzufindender Partikel mit dem für das RMV spezifischen infektiösen Agens selbst erhöht werden.

Nach mechanischer Übertragung des uns zur Verfügung stehenden RMV-Isolats traten folgende Symptome auf: a) auf Zuckerrüben (Sorte Kleinwanzlebener E, Nr. 9819) die für dieses Virus bekannten typischen fleckigen Sekundärsymptome; primär infizierte Blätter zeigen bei beginnender Altersvergilbung auf gelbem Grund dunkelgrüne, in der Regel 0,5 bis $1 \mathrm{~mm}$ breite Ringe mit einem Radius von etwa 3 bis $4 \mathrm{~mm}$; Größenabweichungen von dieser Norm treten je nach Kulturbedingungen mehr oder weniger häufig auf; b) auf Beta patellaris primär und sekundär in der von B e n n e $\mathrm{t} \mathrm{t}^{4}$ beschriebenen Form; c) auf Chenopodium amaranticolor * in Form rotgeränderter Nekrosen als Primärsymptom und sekundär als Blattnekrose nach vorangehender Adernnekrose; die befallenen Pflanzen wachsen nicht selten gesund nach; d) auf Tetragonia expansa * (Neuseeländer Spinat) nur als primäre Chlorosen, die teilweise nekrotisieren; Sekundärinfektion tritt nicht ein.

Werden sekundärkranke Blätter nach der von

1 H. L. $\mathrm{N}$ ix on u. M. A. W a t s o n, Nature [London] 168, 523 [1951].

2 J. Brandes u. K. Zimmer, Phytopathol. Z. 24, 211 [1955].

3 Eigene Arbeiten, noch unveröffentlicht.
Johnson ${ }^{5}$ angegebenen Exsudationsmethode untersucht, so findet man sowohl in Rüben als auch in Chenopodium amaranticolor, in letzterem jedoch in

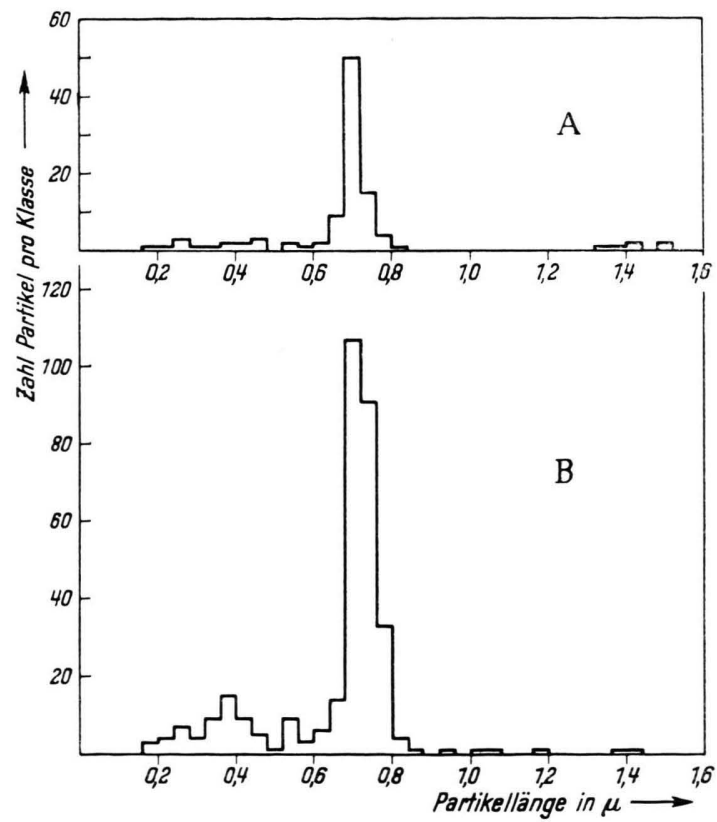

Abb. 2. Längenverteilung des Rübenmosaikvirus: a) im Exsudat aus Rübenblättern, b) im Exsudat aus Blättern von Chenopodium amaranticolor.

größerer Zahl, mehr oder weniger starre, scheinbar wenig flexible Stäbchen (Abb. $1^{* *}$ ). Durch Vermessung von insgesamt 429 Partikeln (98 aus Rüben und 331 aus Chenopodium amaranticolor) wurde die häufigste Länge mit ziemlich genau $700 \mathrm{~m} \mu$ ermittelt (Abb. 2).

4 C. W. B e nnet t, Phytopathology 39, 669 [1949].

* Bislang als Wirtspflanze für RMV nicht beschrieben.

5 J. J o h n s on, Phytopathology 41, 78 [1951].

** Abb. 1 s. Tafel S. 384 b. 
In gesunden Pflanzen waren solche Partikel nicht aufzufinden. Werden Primärinfekte (Nekrosen bei Chenopodium amaranticolor und Chlorosen bei Neuseeländer Spinat) aus den Blättern mit einem Korkbohrer geeigneter Größe ausgestochen und in einem Mikro-Gewebemörser in etwas Wasser homogenisiert, so lassen sich im Überstand die gleichen Partikel nachweisen, sie fehlen jedoch vollständig in dem gesunden, zwischen den primär infizierten Stellen gelegenen Gewebe.

Die oben beschriebene Kombination von Symptomen dürfte das von uns verwendete RMV hinreichend charakterisieren; eine Verwechslung mit einem der anderen auf Rüben vorkommenden bekannten
Viren ist daher ausgeschlossen. Die Tatsache, daß sowohl in den primären Infektionsherden als auch in den Exsudaten sekundärkranker Blätter nur Partikel der abgebildeten Art und keine anderen, einem Virus ähnelnden Teilchen, aufzufinden sind, berechtigt zu dem Schluß, daß die dargestellten Partikel mit einer Länge von $700 \mathrm{~m} \mu$ die die Infektiosität tragenden Einheiten des Rübenmosaikvirus sind.

Wir danken Herrn Prof. Dr. G. S chra m m und Herrn G. B e r g e r im Max-Planck-Institut für Virusforschung, Tübingen, für die Ermöglichung bzw. Durchführung der elektronenmikroskopischen Aufnahmen sowie der Deutschen Forschungsgemein$\mathrm{s} \mathrm{ch}$ a f $\mathrm{t}$ für finanzielle Hilfe.

\title{
Experimentelle Untersuchungen über die Entstehung asexueller Klone bei der Hydromeduse Eleutheria dichotoma
}

\author{
Von C. Hauenschild \\ Aus dem Max-Planck-Irsstitut für Biologie (Abt. H a r t m a n n), Tübingen \\ (Z. Naturforschg. 11 b, 394-402 [1956]; eingegangen am 10. März 1956)
}

\begin{abstract}
Die Hydromeduse Eleutheria dichotoma pflanzt sich normalerweise sowohl geschlechtlich als auch vegetativ fort. In Klonkulturen treten jedoch als Ausnahmen sprunghaft asexuelle Medusen auf, welche die Fähigkeit zur Keimzellenbildung eingebüßt haben und sich nur noch vegetativ fortpflanzen; auch von den am Polypen entstehenden Primärmedusen ist nicht selten ein kleiner Prozentsatz bereits asexuell. In beiden Fällen erscheinen in der vegetativen Nachkommenschaft der geschlechtslosen Medusen, auch bei jahrelanger Zucht, keine sexuell differenzierten Exemplare mehr. Durch Zerstückelung und Aufzucht der Regenerate sowie durch Röntgenbestrahlung ließ sich ein derartiger Verlust der Sexualität z. T. künstlich herbeiführen. Asexuelle Individuen konnten nur durch Implantation von Normalmedusen-Gewebe wieder zur Keimzellenbildung veranlaßt werden; dabei waren die an I-Zellen besonders reichen Implantate am wirksamsten. Dagegen war eine entsprechende Einpflanzung von Polypengewebe stets erfolglos.
\end{abstract}

$\mathrm{M}$ anche niedere Metazoen (vor allem gewisse Schwämme, Hydrozoen, Bryozoen, Turbellarien und Anneliden) vermehren sich hauptsächlich vegetativ, pflanzen sich aber außerdem, sei es regelmäßig oder nur unter bestimmten Bedingungen, auch geschlechtlich fort. In allen bisher bekannten Fällen geht dabei die Potenz des Stammindividuums, Keimzellen zu erzeugen, uneingeschränkt auf alle vegetativen Nachkommen über (von Stockbildung mit polymorpher Differenzierung natürlich abgesehen); auch können bei derartigen Organismen häufig beliebige Teilstücke vollständige Individuen, die nach wie vor die sexuelle Potenz besitzen, regenerieren. Dies beruht sehr wahrscheinlich darauf, daß bei solchen Tieren der ganze Körper von diffus verteilten, undiffe- renzierten Zellen durchsetzt ist (z. B. I-Zellen der Hydrozoen, Neoblasten der Anneliden), welche omnipotent und damit auch imstande sind, sich gegebenenfalls in Keimzellen zu differenzieren. Demgemäß scheint die Fähigkeit zur vegetativen Fortpflanzung die Existenz einer determinierten Keimbahn auszuschließen.

Es sind aber auch Arten bekannt, die sich nur noch vegetativ vermehren und die Sexualität augenscheinlich vollkommen verloren haben. So pflanzt sich der sedentäre Polychaet Ctenodrilus serratus in der Nordsee ausschließlich durch Querteilung fort, während aus Neapel sexuell differenzierte, zwittrige Exemplare beschrieben worden sind. Bisher wurde meist angenommen, daß ein solcher Verlust der Sexualität 